\title{
Painful neuropathy in a diabetic patient resulting from lung cancer and not diabetes: A case report
}

\author{
HE-BIN YAO, YA-NING CHEN, JIAN SHANG and QIAO-JUN HAN \\ Department of Endocrinology, Chinese People's Liberation Army Navy General Hospital, Beijing 100048, P.R. China
}

Received January 24, 2015; Accepted September 21, 2015

DOI: $10.3892 / \mathrm{ol} .2015 .3808$

\begin{abstract}
The current study reports the case of a 61-year-old man with diabetes who was suffering from generalized pain over the whole body and gradually progressive numbness. The patient was initially diagnosed with diabetic peripheral neuropathy and received treatment, however, the symptoms persisted. In October 2010, the patient was admitted to the Chinese People's Liberation Army Navy General Hospital (Beijing, China) for the treatment of diabetes, however, a full-body sharp pain was also described, which was relieved upon massaging the area. Causes, other than diabetes, were investigated for these symptoms. Chest computed tomography and positron emission tomography-computed tomography scans revealed a mass shadow in the right lower lobe of the lung, with multiple lymphatic metastases. Lung cancer was diagnosed with a tumor-node-metastasis stage of T1N3Mx. Following treatment of the cancer with chemotherapy and radiotherapy, the patient's symptoms were significantly improved. The present study reports a rare case of a paraneoplastic neurological syndrome (PNS) that presented as painful neuropathy resulting from lung cancer, which mimicked diabetic peripheral neuropathy.
\end{abstract}

\section{Introduction}

Diabetes is a serious global health burden. It is estimated that the total number of diabetic patients worldwide was $~ 382$ million in 2013, a figure expected to rise to $\sim 592$ million by 2035 (1). Diabetic peripheral neuropathy is a frequent complication of diabetes that $30 \%$ of hospitalized and $20 \%$ of community-dwelling patients with diabetes develop (2). Clinically, peripheral neuropathy patients often presents with symmetrical pain and sensory abnormalities, and numbness

Correspondence to: Dr He-Bin Yao, Department of Endocrinology, Chinese People's Liberation Army Navy General Hospital, 6 Fucheng Road, Haidian, Beijing 100048, P.R. China

E-mail: yhb196321@163.com

Key words: diabetic peripheral neuropathy, lung cancer, paraneoplastic neurological syndrome starting from the distal end of the lower limb is often the early typical symptom. Painful diabetic peripheral neuropathy is present in $20 \%$ of patients with type 2 diabetes and $5 \%$ of those with type 1 (3). Such patients often feel a prickling pain, a burning pain, or a drilling and cutting pain, and they may also present with hyperalgesia. The correct diagnosis of painful diabetic peripheral neuropathy is made only after other causes, besides diabetes mellitus, are ruled out (4). The diagnosis depends upon the corresponding symptoms and signs, the results from nerve conduction studies, monofilament and tuning fork examination results, temperature perception and ankle reflex evaluation results (5-7). The treatment mainly includes achieving satisfactory blood glucose control, improving microcirculation, neurotrophic drugs and other symptomatic treatment to improve symptoms. Patients with type 2 diabetes have an increased risk of developing cancer, and the association between diabetes and cancer is currently a popular topic (8). The present study reports a rare case of lung cancer that presented with paraneoplastic neurological syndrome (PNS), which mimicked diabetic peripheral neuropathy.

\section{Case report}

In September 2010, a 61-year-old man with diabetes mellitus, coronary heart disease, hypertension and pulmonary fibrosis presented at Suzhou People's Hospital (Suzhou, China), with a sensory disturbance, and the feeling of pins and needles all over the body. The patient presented with a 1-year history of type 2 diabetes, which was being treated with $500 \mathrm{mg}$ metformin twice a day and $2.0 \mathrm{mg}$ glibenclamide twice a day for $\sim 9$ months. In August 2010, a chest computed tomography scan was performed to test for pulmonary fibrosis, and no other symptom except for pulmonary fibrosis was reported. Treatment with tramadol was administered for diabetic peripheral neuropathy, but the patient's condition did not improve. Following referral to the pain clinic of Peking Union Medical College Hospital (Beijing, China), the patient was found to be negative for antinuclear antibodies. Experimental treatment with prednisone acetate $10 \mathrm{mg}$ once a day for 4 days was attempted, but the symptoms progressed, such that the patient experienced difficulty in sleeping and lost $5 \mathrm{~kg}$ in weight. The patient was therefore admitted to the Chinese People's Liberation Army Navy General Hospital (Beijing, China) for examination and treatment in October 2010. 


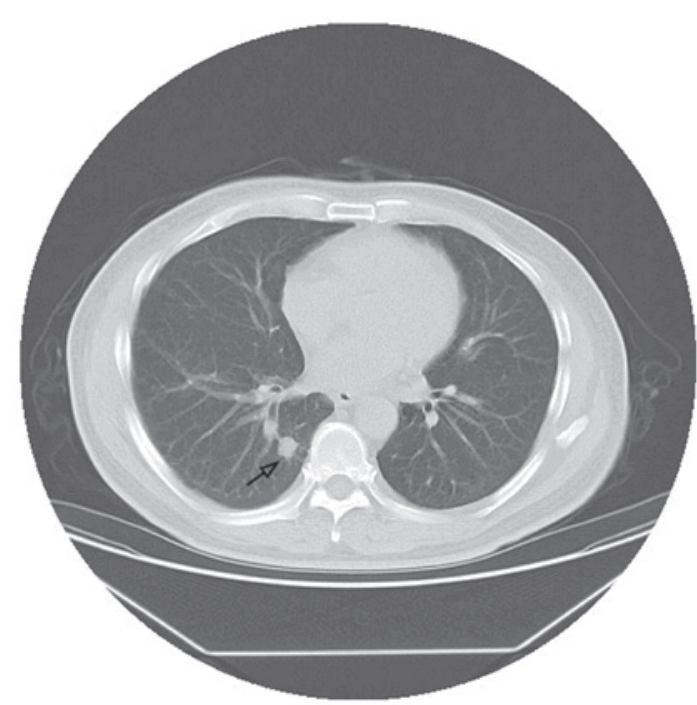

Figure 1. Computed tomography showing a mass shadow close to the spine (arrow).

Upon presentation, the patient complained of full-body pain and numbness, and massage was found to relieve this pain. The patient scored the pain as 10 out of 10 for severity. Cardiovascular, respiratory, abdominal and musculoskeletal examinations were normal. Scattered skin rashes were present on the patient's back. A neurological examination showed that the muscle tone was normal, the bilateral tendon reflexes were decreased and the bilateral Babinski signs were negative. Blood testing revealed the following: Fasting glucose, $11.7 \mathrm{mmol} / 1$ (normal range, 3.9-6.1 mmol/1); glycated hemoglobin (HbA1c), 9.7\% (normal range, 3.0-6.3\%); serum albumin, $28 \mathrm{~g} / 1$ (normal range, 34.0-48.0 g/l); total protein, $58 \mathrm{~g} / 1$ (normal range, 60.0-83.0 g/l); erythrocyte sedimentation rate, $52 \mathrm{~mm} / \mathrm{h}$ (normal range, $0.00-15.0 \mathrm{~mm} / \mathrm{h}$ ); and C-reactive protein, $16.4 \mathrm{mg} / \mathrm{l}$ (normal range, 0.0-8.2 mg/l). The serum creatine kinase, creatine kinase-MB, full blood count and renal, hepatic and autoimmune profiles were all normal.

A primary diagnosis of diabetic peripheral neuropathy was formed and biosynthetic human insulin injection was administered before three meals by subcutaneous injection, and isophane protamine biosynthetic human insulin injection was administered before bed, to treat the hyperglycemia. Thioctic acid for injection $0.6 \mathrm{~g}$ once a day for 7 days and analgesics (200 mg carbamazepine once a day before bed and $100 \mathrm{mg}$ tramadol twice a day for 10 days) were used to relieve the diffuse pain. However, there was no improvement in the symptoms. The patient then reported dizziness when getting up in the morning, and the recorded arterial blood pressure dropped $40 \mathrm{mmHg}$ for the systolic blood pressure and $20 \mathrm{mmHg}$ for the diastolic blood pressure when the patient stood up. Orthostatic hypotension was diagnosed. The results from a motor nerve electromyography and retinopathy were normal, and the urinary albumin level was in the normal range. The paradoxical phenomenon of severe symptoms and a lack of any detectable cause suggested that the pain may not be caused by the diabetes. A computed tomography scan of the chest subsequently revealed an enlarged node in the lower right lung close to the spine (Fig. 1). On positron emission tomography-computed tomography, the focus nodes

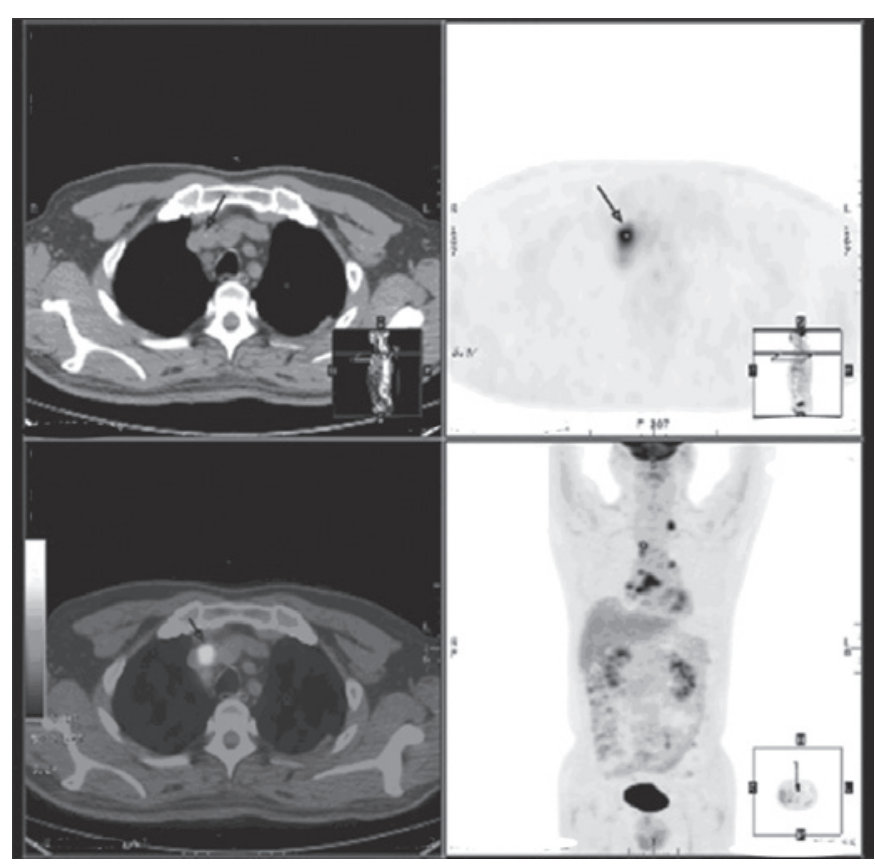

Figure 2. Positron emission tomography showing tumor-specific uptake in a region other than the lung (arrow).

of tumor-specific uptake were also observed in regions other than the lung (Fig. 2). The tumor-node-metastasis stage of the lung tumor was $\mathrm{T} 1 \mathrm{~N} 3 \mathrm{Mx}(9)$. The patient rejected invasive examinations, such as a lymph node biopsy. However, based on the aforementioned results, the patient was diagnosed with sensory neuropathy resulting from PNS.

Following 6 months of chemotherapy and radiotherapy, the symptoms were gradually reduced, but only carbamazepine could relieve the patient's pain. In May 2012, the serum albumin, total protein, erythrocyte sedimentation rate and C-reactive protein levels returned to normal, however, the pain was still being relieved with an analgesic.

\section{Discussion}

Peripheral polyneuropathy is a frequent complication of diabetes (10). Approximately $30 \%$ of diabetic patients develop neuropathic pain, which manifests as spontaneous pain, hyperalgesia and allodynia (11). Several studies have identified other potential causes of neuropathy in individuals with diabetes (4). A total of $10-50 \%$ of individuals with diabetes and peripheral neuropathy may have a separate cause of peripheral neuropathy, including neurotoxic medications, vitamin B12 deficiency, alcohol abuse, chronic inflammatory demyelinating neuropathy, renal disease, inherited neuropathy and vasculitis, and certain patients may even have more than one of these causes (4). Paraneoplastic neuropathies are also a potential cause (12).

The patient in the present study did not display the classical characteristics of diabetic neuropathy, such as hyperalgesia and allodynia, which was brought on by the light pressure of wearing socks, stockings or bedclothes, but displayed unusual symptoms, such as pain that could be relieved by massage. Additionally, the patient did not display clinical features of diabetic retinopathy or diabetic kidney 
disease and therefore, the diagnosis of diabetic neuropathy was not supported (12). The patient exhibited the characteristics of classical paraneoplastic sensory neuropathy, which corresponded to the following criteria (13): Sub-acute development in $<12$ weeks, a Rankin severity score of $\geq 3$ (moderate disability), the onset of numbness with frequent pain and sensory disturbance, affected arms and legs (the so-called gloves and stockings areas), and a frequently asymmetrical onset (13). The diagnosis of paraneoplastic peripheral neuropathies should be considered in this patient, even though it is a rare neuroimmunological complication of malignancy. The manifestation of paraneoplastic sensory neuropathy is restricted to the peripheral nervous system in $24 \%$ of patients. The tumors that are typically associated with these syndromes are lymphoma, thymoma, and breast, ovarian and small cell lung carcinoma. The most common autoantibody marker of paraneoplastic neuropathies is anti-Hu (also known as type 1 anti-neuronal nuclear antibody).

In the current patient, lung cancer was found 2 months after the characteristics of classical paraneoplastic sensory neuropathy occurred. The patient's pain was significantly improved following treatment for lung cancer without concomitant immunotherapy. PNS was diagnosed based on the diagnostic criteria established for the disease by an international panel of neurologists $(14,15)$. However, paraneoplastic antibodies were not detected in the serum or pathological samples.

This case highlights the importance of forming a differential diagnosis that considers conditions other than diabetic complications in diabetic patients who present with neurological symptoms, particularly in the presence of non-typical features, such as generalized aching. Diabetes should not be assumed to be the only cause of painful neuropathy in diabetic patients.

\section{Acknowledgements}

The authors would like to thank Edanz for providing an English editing service.

\section{References}

1. Guariguata L, Whiting D, Hambleton I, Beagley J, Linnenkamp U and Shaw J: Global estimates of diabetes prevalence for 2013 and projections for 2035. Diabetes Res Clin Pract 103: 137-149, 2014.

2. Vinik AI and Casellini CM: Guidelines in the management of diabetic nerve pain: Clinical utility of pregabalin. Diabetes Metab Syndr Obes 6: 57-78, 2013.

3. Hartemann A, Attal N, Bouhassira D, Dumont I, Gin H, Jeanne S, Said G and Richard JL; Working Group on the Diabetic Foot from the French-speaking Society of Diabetology: Painful diabetic neuropathy: Diagnosis and management. Diabetes Metab 37: 377-388, 2011.

4. Freeman R: Not all neuropathy in diabetes is of diabetic etiology: Differential diagnosis of diabetic neuropathy. Curr Diab Rep 9: 423-431, 2009.

5. Hirschfeld G, von Glischinski M, Blankenburg $M$ and Zernikow B: Screening for peripheral neuropathies in children with diabetes: A systematic review. Pediatrics 133: e1324-e1330, 2014.

6. Shehab DK, Al-Jarallah KF, Abraham M, Mojiminiyi OA, Al-Mohamedy $\mathrm{H}$ and Abdella NA: Back to basics: Ankle reflex in the evaluation of peripheral neuropathy in type 2 diabetes mellitus. QJM 105: 315-320, 2012.

7. Feng Y, Schlösser FJ and Sumpio BE: The Semmes Weinstein monofilament examination as a screening tool for diabetic peripheral neuropathy. J Vasc Surg 50: 675-682, 2009.

8. Zhang PH, Chen ZW, Lv D, Xu YY, Gu WL, Zhang XH, Le YL, Zhu HH and Zhu YM: Increased risk of cancer in patients with type 2 diabetes mellitus: A retrospective cohort study in China. BMC Public Health 12: 567, 2012.

9. Wohlschläger J, Wittekind C and Theegarten D: New TNM classification of malignant lung tumours. Pathologe 31: 355-360, 2010.

10. Azulay JP and Pouget J: Diabetic neuropathies. Rev Prat 51: 1783-1787, 2001 (Article in French).

11. Wang D, Couture R and Hong Y: Activated microglia in the spinal cord underlies diabetic neuropathic pain. Eur J Pharmacol 728: 59-66, 2014.

12. Tesfaye S, Vileikyte L, Rayman G, Sindrup SH, Perkins BA, Baconja M, Vinik AI and Boulton AJ; Toronto Expert Panel on Diabetic Neuropathy: Painful diabetic peripheral neuropathy: Consensus recommendations on diagnosis, assessment and management. Diabetes Metab Res Rev 27: 629-638, 2011.

13. Kanaji N, Watanabe N, Kita N, Bandoh S, Tadokoro A, Ishii T, Dobashi $\mathrm{H}$ and Matsunaga T: Paraneoplastic syndromes associated with lung cancer. World J Clin Oncol 5: 197-223, 2014.

14. Honnorat J and Antoine JC: Paraneoplastic neurological syndromes. Orphanet J Rare Dis 2: 22, 2007.

15. Graus F, Delattre JY, Antoine JC, Dalmau J, Giometto B, Grisold W, Honnorat J, Smitt PS, Vedeler Ch, Verschuuren JJ, et al: Recommended diagnostic criteria for paraneoplastic neurological syndromes. J Neurol Neurosurg Psychiatry 75: 1135-1140, 2004. 